\title{
Signal modulation techniques in non-orthogonal waveform for future wireless communication system
}

\author{
Siti Rosmaniza AR ${ }^{1}$, Norulhusna Ahmad ${ }^{2}$, Sharifah Kamilah Syed Yusof ${ }^{3}$ \\ ${ }^{1,3}$ School of Electrical Engineering, Faculty of Engineering, Universiti Teknologi Malaysia, Malaysia \\ ${ }^{2}$ Razak School of Engineering and Advanced Technology, Universiti Teknologi Malaysia, Malaysia \\ ${ }^{1}$ Fakulti Kejuruteraan Elektronik dan Kejuruteraan Komputer, Universiti Teknikal Malaysia Melaka, Malaysia
}

\begin{tabular}{l} 
Article Info \\
\hline Article history: \\
Received Jan 20, 2019 \\
Revised Mar 5, 2019 \\
Accepted May 3, 2019 \\
\hline
\end{tabular}

Keywords:

Accumulator

Bit error rate

Non-orthogonal waveform

Signal modulation

\begin{abstract}
In the future generation of the wireless communication system, the utilization of bandwidth needs to be managed efficiently. Due to that, research on enhancing the efficiency of the bandwidth has recently attracted numerous interests focusing more on the non-orthogonal waveform generation and detection. Even though non-orthogonal waveform is promising a higher efficiency, it introduces inter-carrier interference (ICI) at the transmitter due to the subcarrier overlapping. In this paper, the signaling technique for nonorthogonal waveform generation and detection is discussed. This paper also proposed the use of accumulator (ACC) to improve the system performance of the signal modulation in the non-orthogonal waveform for the next generation of the wireless communication system. The result is represented in an EXtrinsic Information Transfer (EXIT) chart to show the effects of adding ACC into the non-orthogonal system.
\end{abstract}

Copyright $@ 2019$ Institute of Advanced Engineering and Science. All rights reserved.

\section{Corresponding Author:}

Siti Rosmaniza Ab Rashid,

School of Electrical Engineering, Faculty of Engineering,

Universiti Teknologi Malaysia,

81310 UTM Johor Bahru, Johor, Malaysia.

Email: sitirosmaniza@utem.edu.my

\section{INTRODUCTION}

The fifth generation (5G) mobile communication system was first released on paper in 2014 and is expected to be entirely deployed in the year 2020 [1]. The new network system promises to provide users with a super high-speed network, higher reliability and lower latency compared to the current engaged system. 5G is the future technology that requires connections of everything which is so-called as Machine-toMachine (M2M) communications. Due to that, 5G will necessitate an innovation in technology especially in the wireless transmission and network system where an advanced in the current technology such as multiple access, multi-antenna, encoding modulation, and even a new waveform design should be deliberated. This new requirement is considered as a significant issue in building future networks with the increase in IP traffic along with the rise of user-oriented mobile multimedia applications. Consequently, there will be an increasing demand for efficient spectral and energy, greater network capacity and higher data rates required along with the increment.

One major improvement that needs to be considered is towards the waveform technology that can better support the $5 \mathrm{G}$ demand and meet the application scenarios of abundant connection instead of mobile broadband connection only [2]. The waveform also needs to have the good scalability to cater the continuous emergence of new technologies and services and to be compatible with other technical ideas such as modulation encoding multiple access technologies, the use of a large-scale antenna and the huge connection of multiple devices. 
Current waveform technology of wireless communication system in $4 \mathrm{G}$ is using Orthogonal Frequency Division Multiplexing (OFDM) technique because of its robustness against the multipath channel. However, recent development in the future network requires less power consumption that makes the orthogonality of the OFDM is restricted due to its synchronization process that consumes much power [3]. OFDM also produces high spectral leakage and a high peak to average power ratio (PAPR) that may affect the high-power amplifier efficiency. Furthermore, OFDM waveform suffers from a high level of out-of-band (OOB) emissions where it leads to higher interference between adjacent channels in the network [4]. Another important finding was that the OFDM system consumes high bandwidth when transmitting at the higher bit rate [5].

Due to the limitation mentioned, new signaling techniques that can generate a more flexible waveform for a convenience future multiservice of a communication system is apparently needed. In conjunction with the necessity, the technique of non-orthogonal signaling is actively studied by many researchers. Most of the recent research agreed that the non-orthogonal for multicarrier communication system could improve the deficiency in the OFDM system where the spectral efficiency of the system can be improved within the same boundary of bandwidth [6]. The non-orthogonal concept for the multicarrier system is introduced by massively overlapping the subcarrier with violation towards orthogonality rules. The non-orthogonal technique is believed to provide optimal use of available bandwidth. The non-orthogonal approach offers an advantage over the orthogonal ones in spectral power efficiency. Besides, by overlapping the subcarrier in the non-orthogonal waveform, it can provide a higher capacity compares to the orthogonal within the same bandwidth preserved.

This paper presents the available non-orthogonal signaling techniques that are currently being studied by many researchers to find the best suitable waveform which can be implemented in the next generation of the wireless communication system. The rest of the paper is organized as follows. Section 2 reviews the basic concept of orthogonal and non-orthogonal signaling technique. In Section 3 we give insights into the proposed enhancement on the detection system while Section 4 is on the results and analysis discussion. Finally, Section 5 summarizes and concludes this survey.

\section{ORTHOGONAL AND NON-ORTHOGONAL WAVEFORM}

The orthogonal waveform is being adopted in the current 4G Long Term Evolution (LTE) communication for the multicarrier transmission system. The waveform system previously used in many other systems also such as Wireless Local Area Network (WLAN), Worldwide Interoperability for Microwave Access (WiMAX) and other broadcasting technologies such as Digital Video Broadcasting (DVB) and Digital Audio Broadcasting (DAB) [7]. So-called as OFDM, it is a form of transmission that uses a large number of orthogonal carriers so that the subcarriers will not interfere with each other. That makes OFDM a chosen modulation technique as it is resilient to the interference especially in the multipath channel. In the OFDM system, each symbol is transmitted with a rectangular pulse of length $T$ and frequency separation, $\Delta f$ for each subcarrier is equal to 1 to ensure that the symbols can be recovered without any distortion. Therefore, the OFDM signal is represented as.

$$
x(t)=\frac{1}{\sqrt{T}} \sum_{m=0}^{\infty} \sum_{k=0}^{K-1} s_{k, m}(t) \exp (j 2 \pi k \Delta f(t-m T))
$$

where $s_{k, m}(t)$ represents the continuous signal of the $k$-th subcarrier in $m$-th OFDM symbol period, $K$ is the number of subcarriers and $T \Delta f=1$.

\subsection{Non-orthogonal Signal Generation}

A non-orthogonal signal is introduced due to the incompatibility of the orthogonal signaling system to adapt to the excessive use of mobile devices for a future wireless communication system. The use of nonorthogonal is proposed for future wireless communication due to its ability to increase the capacity and bandwidth efficiency via massive overlapping of the subcarrier. The difference of orthogonal and nonorthogonal is depended on the relationship of frequency separation, $\Delta \mathrm{f}$ of the system with length, $\mathrm{T}$ where the orthogonality is violated when $\mathrm{T} \Delta \mathrm{f} \neq 1=\alpha$.

Some researchers had proposed a variety of new multicarrier technology that practices the nonorthogonal concept. The signal generation and detection of those non-orthogonal signaling mainly involved three different technique which is basically on the (i) filter-bank based, (ii) linear modulation based and (iii) Fourier Transform (FT) based. 


\subsubsection{Filter-Bank Technique}

The signal process in the filter bank technique is completed by using an array of filter that separates the input signal into multiple components where each of the original signals will carry the single frequency sub-band. According to [8], the use of a filter bank technique is an alternative transmission method that can help in avoiding the embarking and emerging noise using high-quality filters. This filter bank technique can be categorized into two types of filtering technique which is on a subcarrier basis for e.g.: Generalized Frequency Division Multiplexing (GFDM) and Filter Bank Multicarrier (FBMC) and on a sub-band basis, for e.g.: Universal Filter Multicarrier (UFMC) and Resource-Block Filtered OFDM (RB-F-OFDM) [9]. GFDM is claimed can enhance the spectral efficiency with the use of tail biting technique to reduce the length of the transmitted symbol compared to using the conventional cyclic prefix (CP) [10]. The input signal is consisting of $K$ independent subcarrier and $M$ number of sub symbols as $d_{k, m}$. Then, the subcarrier is filtered individually by using a prototype filter, $G$ (i.e.: pulse shaping, circular shifted etc.) to generate a nonorthogonal signal $x[n]$ as.

$$
x[n]=\sum_{k=0}^{K-1} \sum_{m=0}^{M-1} g_{k, m}[n] d_{k, m}
$$

where $g_{k, m}$ is the transmit filter with,

$$
g_{k, m}[n]=g[(n-m K) \bmod N] \exp \left(-j 2 \pi \frac{k}{K} n\right)
$$

with $n$ is the time sample index where modulo operations cause shifting operation in frequency complex exponential.

In FBMC, the subcarriers are filtered through an array of filters, but it can transmit at a higher bit rate and more efficient than OFDM because no CP added to the system [11]. The FBMC system generates signal with the parallel transmit filter known as Synthesis Filter Bank (SFB) [12]. The first block in OQAM pre-processing is the complex-to-real conversion block that separates the imaginary and real parts of the complex symbols, $\boldsymbol{C}_{K, m}=\left[c_{0, m}, c_{1, m}, \ldots c_{K-1, m}\right]$ to form a new symbols of $\boldsymbol{d}_{K, m}=\left[d_{0, m}, d_{1, m}, \ldots d_{K-1, m}\right]$. The symbol is multiplied with a sequence of $\boldsymbol{\theta}_{K, m}=\left[\theta_{0, m}, \theta_{1, m}, \ldots \theta_{K-1, m}\right]$ where $\theta_{k, m}=\exp \left(j \frac{\pi(k+m)}{2}\right)$. The signal is then forwarded to SFB where the up-sampling process is done before filtering with the selected prototype filter. Later, all the sub-signals are summed altogether to create the output of the signal generation in the FBMC transmission system as in (3) instead that the basis pulse $g_{k, m}(t)$ is define as [13].

$$
g_{k, m}(t)=p(t-m T) e^{j 2 \pi k F(t-M T)} e^{j \frac{\pi}{2}(k+m)}
$$

where $p(t)$ is the proptotype filter.

Meanwhile, UFMC and RB-F-OFDM use the concept of sub-band filtering where the signal is filtered over the entire band. The idea of grouping the subcarriers provides an advantage to the UFMC where it can reduce the filter length compared to the FBMC [14].

In UFMC, the subcarriers were divided into sub-bands where each sub-band had a fixed number of subcarriers and was filtered by a filter length of $L$. At the channel, the response from all the filter is being summed up for the transmission. The signal generation for the UFMC system is shown as:

$$
x[n]=\sum_{k=1}^{B_{i}} \sum_{n=0}^{N_{k}-1} s_{i}^{k}(n) e^{j 2 \pi n \Delta f t} F_{i}^{k}\left(t-n T_{d}\right)
$$

where $s_{i}^{k}(n)$ is the data vector of the $i$-th user in the $k$-th sub-band, $F_{i}^{k}$ is the filter function and $\Delta f=1 / T_{d}$ is the subcarrier spacing.

\subsubsection{Linear Modulation Technique}

FTN is focused on the provision of the next-generation wireless system because of its effectiveness in improving spectral efficiency with its faster sampling process compared to the Nyquist rate [15], [16]. FTN is able to transmit 30-100\% more data in the same bandwidth within the same transmit power and error rate compared to the current method [17]. Generally, a transmitted FTN signal over the channel is merely a linear modulation form that can be represented as.

$$
x(t)=\sum_{m=0}^{M-1} a_{m} P(t-m \rho T)
$$

where $a_{m}$ is the data symbols, $P(t)$ is the baseband pulse and $\rho$ is range from 0 to 1 , where $\rho=1$ is producing an orthogonal waveform. 


\subsubsection{Fourier Transform Technique}

Fourier Transform (FT) is the main technique involved in the OFDM system where it applies the concept of Inverse Discrete Fourier Transform/Discrete Fourier Transform (IDFT/DFT). This technique is used due to its easy signal conversion from the frequency domain to the time domain. For the concept of the non-orthogonal waveform, the technique in OFDM is maintained, but the orthogonality is violated so that it can improve more on the spectral efficiency within the preserved bandwidth.

The non-orthogonal in the FDM system that implements IDT/DFT were introduced by Hamamura and Tachikawa (2004) with its multicarrier transmission name as HC-MCM [18]. The idea later being extended with the technique called Spectral Efficiency Frequency Division Multiplexing (SEFDM) [19] where the signal generation process is applied using a filter bank technique [20]. However, the system is improved by applying IDFT so that a system developed will lead to better performance with a lower computational complexity [21]. In Overlapped FDM (OvFDM) or Non-Orthogonal FDM (NOFDM), the subcarriers are overlapped, and this made the effective bandwidth of the subcarriers become larger than the frequency coherent bandwidth [22]. Thus, it will help in enhancing the system performance for the nonorthogonal system. The next following section will explain more on the signal detection that is proposed as a potential technique in detecting and decoding the signal received at the receiver

\subsection{Non-Orthogonal Signal Detection}

Signal detection for non-orthogonal system involves the process of decoded, detection and equalization. Most of the system mentioned in Section III use the same detection and equalization method. There are few types of equalization such as linear equalizer, decision feedback equalizer, and an adaptive equalizer. The equalizer functions to reverse the distortion occurs when a signal is transmitted within a channel. A linear equalizer such as Zero Forcing (ZF) and Minimum Mean Square Error (MMSE) process the incoming signal using a linear filter. ZF techniques force the interference to be zero while MMSE minimizes the total power of interference by measuring the output between the equalizer and the transmitted symbol. However, the use of ZF at the same time increase the noise while MMSE only reduce the total power of the noise. Therefore, MMSE and ZF may contribute to the higher noise increment due to the ICI occurrence in the non-orthogonal system [23].

However, due to the massive overlapping created in the non-orthogonal system, this creates a severe interference and higher noise enhancement which requires a superior detection system [5]. The use of linear equalization is no longer applicable, and suggestions on the turbo equalization together with the hybrid technique had risen as a potential detector where the performance of the turbo equalization is gradually improved by the iterative concept. Turbo equalization is based on the Maximum-a-Posteriori (MAP) or Viterbi algorithm (VA) and Bahl-Cocke-Jelinek-Raviv (BCJR) algorithm. However, the computational complexity of MAP principles increases with the increased memory length and in larger constellation sizes [5].

VA allows safe data transmission and can help to recover signals by correcting errors, and it is most reliable for single bit error detection and correction. However, for the error detection and correction in the non-orthogonal system requires the VA to work by combining with other detection technique efficiently. Some researcher proposes the use of Maximum Likelihood Sequence Estimation/Detection (MLSE/D). Maximum Likelihood Sequence Estimation/Detection (MLSE/D) estimates the sequence of the transmitted symbol by choosing the transmitted symbol of the input sequence in a way to produce the maximum likelihood function of the signal received. The SEFDM system and FTN system apply the use of MLSE/D in detection and equalization of the system to minimize the probability of error in the system. However, the use of MLSE/D in the non-orthogonal system gaining a higher computational complexity for the system in mitigating an increased ISI [24].

Therefore, a few combination techniques are proposed to achieve a better spectral enhancement and reduce the complexity of the system implementation. For instance, in [25] has proposed a turbo equalizer namely soft-cancellation minimum mean square error (SC-MMSE). The technique is functioned as severe ICI mitigation for the non-orthogonal system. The technique shows a higher bandwidth efficiency can be achieved closer to the theoretical limit. A combination of MMSE-ML scheme is also proposed for SEFDM system in [23] to fight the poor performance of MMSE alone and computational complexity of ML alone and achieve a near optimum error performance with polynomial complexity. While in FTN detection, a combination of semidefinite relaxation and sequence estimation which is known as generalized approach semidefinite relaxation (SDR)-based sequence estimation (GASDRSE) and set theory SDR-based sequence estimation (STSDRSE) technique is proposed in [26]. This technique shows an increase in data rate and higher spectral efficiency. However, most of the implementations for the detection system is restricted to a free-ISI condition only. 


\section{ENHANCEMENT IN NON-ORTHOGONAL SIGNAL DETECTION}

In realizing the $5 \mathrm{G}$ networking system, many targets need to be achieved to support the diversified usage scenarios in the next generation of massive Internet of Things (IoT) system. For instance, the need for advanced new radio transmission technologies such as new waveform and new channel coding is required in achieving the goal.

\subsection{System Model}

In [25] and [27], the author has proposed a non-orthogonal system known as Generalized Inverse Discrete Fourier Transform (GIDFT-nOFDM) which has higher subcarrier compare to a total number of sampling. The system block diagram is shown in Figure 1, and its transmitted signal is represented as:

$$
x[t]=\frac{1}{\sqrt{T}} \sum_{m=-\infty}^{\infty} \sum_{n=0}^{N_{b}-1} S_{n, m} e^{j 2 \pi n \Delta f(t-m T)}
$$

where $N_{b}$ is the total number of subcarriers, $s_{n, m}$ is the $n t h$ subcarrier in the $m t h$-OFDM symbol, $\Delta f$ is the frequency separation as define in (1). For this GIDFT matrix to achieve the massive overlapping concept, the value of $\alpha$ must be less than one and it is represented as

$$
\alpha=\frac{N_{c}}{N_{b}}
$$

where $N_{c}$ is the total number of sampling. By having $N_{c}$ less than the number of $N_{b}$, bandwidth can be efficiently utilized.

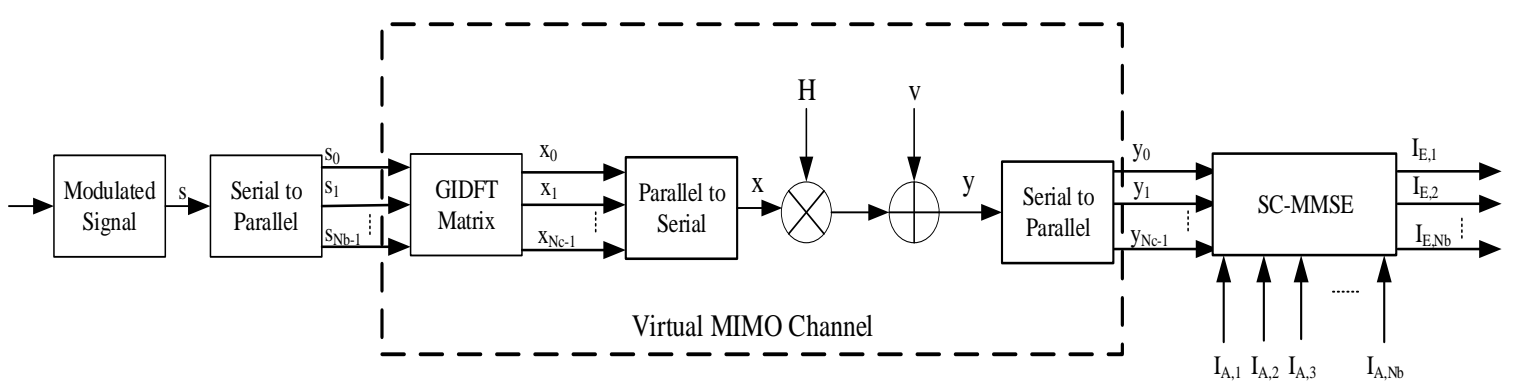

Figure 1. GIDFT-nOFDM system [25]

The GIDFT matrix is being multiplexed with the modulated signal that will produce the received signal as.

$$
y=H x+v
$$

And,

$$
\mathrm{x}=\mathrm{G}_{\mathrm{N}_{\mathrm{C}}} \mathrm{S}
$$

where $y=\left[y_{o}, y_{1}, \ldots, y_{N_{C}-1}\right]^{T}$ is the received signal vector, $\mathrm{H}$ is the channel, $S=\left[s_{0}, s_{1}, \ldots, s_{N_{b}-1}\right]^{T}$ is the input signal vector, and $v=\left[v_{0}, v_{1}, \ldots, v_{N_{C}-1}\right]^{T}$ is the noise vector. $G_{N_{C}}$ is the GIDFT matrix where the matrix elements with $k=0,1, \ldots, N_{C}-1$ and $n=0,1, \ldots, N_{b}-1$ is given as

$$
g_{k, n}=\frac{1}{\sqrt{N_{c}}} \exp \left(\frac{j 2 \pi k n}{N_{b}}\right)
$$

As shown in Figure 1, the system model where GIDFT is considered as the virtual Multiple-InMultiple-Out (MIMO) channel to provide simplification towards the system. Instead, the receiver of the system is proposed with the use of soft-cancellation minimum mean-squared error (SC-MMSE) turbo equalization to solve the severe noise occurrence due to higher overlapping among the subcarriers. 


\subsection{Proposed System Enhancement}

As mentioned in [25], the author represents an EXtrinsic Information Transfer (EXIT) chart [28] to analyze the matching between the BCJR decoder and equalizer for a recursive system. However, the results showed that the ending point is not exactly reached $(1.0,1.0)$ point which makes the system unable to eliminate the error floor. In order to enhance the system performance of [25], a doped accumulator (ACC) is proposed to eliminate the BER floor where it is placed between the interleaver and the modulation of the system at the transmitter. Figure 2 shows the diagram of the proposed ACC in an example of rate-1 Recursive Systematic Convolutional Code (RSCC) and its condition is given as in (12)

$$
\begin{gathered}
\hat{b}_{k}=\widehat{b_{k-1}} \oplus b^{\prime}{ }_{k} \\
\widetilde{b_{k}}=\left\{\begin{array}{l}
b^{\prime}{ }_{k} \text { for } \bmod _{p}(k) \neq 0 \\
\widehat{b_{k}} \text { for } \bmod _{p}(k)=0
\end{array}\right.
\end{gathered}
$$

where $k$ is the bit-timing index, $\hat{b}_{k}$ is the ACC output bit, $\widetilde{b_{k}}$ is the channel encoded bit and D is the delay. Due to the use of recursive receiver, a doped de-accumulator needs to be added to the receiver system so that the bits received can be correctly detected and decoded.

\section{RESULT AND ANALYSIS}

Based on Figure 2, the system model is proposed to receive the series sequence signal from the GIDFT matrix to be processed in the SC-MMSE blocks equipped with the ACC blocks to enhance further the rate of error received in the system. The analysis in [25] shows that the EXtrinsic Information Transfer (EXIT) chart [28] that was used to analyze the matching between the BCJR decoder and the equalizer of the recursive system does not exactly reach $(1.0,1.0)$ point which makes the system unable to eliminate the error floor.

Figure 3 shows the EXIT chart for the GIDFT-nOFDM system with and without the existence of ACC at the various value of overlapping factor, $1-\alpha$ versus the BCJR decoder with $S N R=5 d B$ and $N_{C}=$ 4. The bigger value of $1-\alpha$ indicates higher overlapping factor which means more bandwidth can be utilized. As the $1-\alpha$ increase, the gap between the EXIT curve of decoder and equalizer become smaller. At certain number of iterations at receiver, the BER turbo cliff with error floor is expected at $S N R=5 d B$ when the overlapping factor $1-\alpha=0.78$. To remove the error floor, the ending point of equalizer EXIT curve need to be pushed to $(1.0,1.0)$ point and this can be achieved by adding doped accumulator. However, the EXIT curve of $1-\alpha=0.78$ is intersect with the BCJR decoder and minimum BER cannot be reached. Better performance of the proposed system at $S N R=5 d B$ can only be obtained by reducing the overlapping factor, and for this case, $1-\alpha=0.7$.

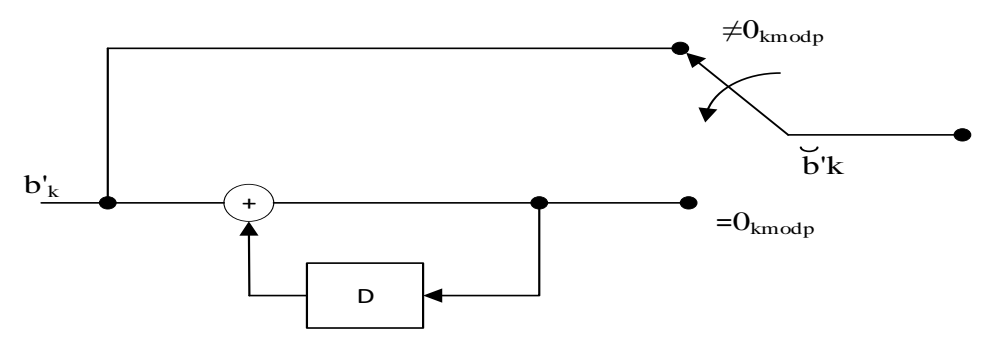

Figure 2. Proposed Rate-1 RSCC doped accumulator at the transmitter

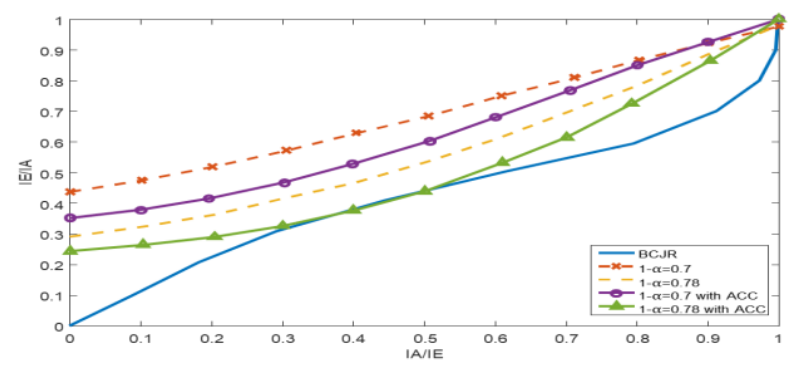

Figure 3. EXIT chart for different overlapping factor, $1-\alpha$ at $\mathrm{SNR}=5 \mathrm{~dB}$ 


\section{CONCLUSION}

This paper is intended to spread the knowledge of non-orthogonal signaling concept which is in demand to be implemented in the 5th generation wireless communication system soon. The scope of this paper is to introduce the technique of signal generation and detection among the non-orthogonal waveform candidate that is currently being studied among the researchers in making the waveform contenders a successful scheme in ensuring a reliable transmission system with an enhancement in bandwidth efficiency is achieved. This paper also proposed the use of ACC to achieve better BER performance even when the system is massively overlapping. Due to that, this system has a good background to be explored more towards real channel of the future wireless communication system.

\section{ACKNOWLEDGEMENTS}

The authors would like to thank the Ministry of Education (MOE) Malaysia, Faculty of Electronic and Computer Engineering (FKEKK), Universiti Teknikal Malaysia Melaka (UTeM) and Razak Encouragement Grant: R.K130000.7740.4J300, Razak Faculty of Technology and Informatics, UTM for sponsoring this research study.

\section{REFERENCES}

[1] ITU, "IMT Vision - Framework and overall objectives of the future development of IMT for 2020 and beyond," 2015.

[2] Y. Ning, "Research on New Multicarrier Transmission Technologies of 5G Research on New Multicarrier Transmission Technologies of 5G," 2017.

[3] N. Michailow et al., "Generalized frequency division multiplexing for 5th generation cellular networks," IEEE Trans. Commun., vol. 62, no. 9, pp. 3045-3061, 2014.

[4] Mohamed Elkourdi, B. Peköz, E. Güvenkaya, and H. Arslan, "Waveform Design Principles for 5G and Beyond," 2016 IEEE 17th Annu. Wirel. Microw. Technol. Conf., 2016.

[5] N. Ahmad, "Non-orthogonal frequency division multiplexing with turbo equalization," 2014.

[6] Y. Wang and Y. Wang, "Capacity analysis and waveform design of a non-orthogonal waveform superposition scheme for high reliable communications," Proc. - IEEE Symp. Comput. Commun., vol. 2016-Augus, pp. 10721077, 2016.

[7] I. Poole, "OFDM : Orthogonal Frequency Division Multiplexing," https://www.radio-electronics.com/, 2014. [Online]. Available: https://www.radio-electronics.com/.

[8] B. Farhang-Boroujeny, "Filter Bank Multicarrier Modulation : A Waveform Candidate for 5G and Beyond," Adv. Electr. Eng., vol. 2014, p. 25, 2014.

[9] M. Van Eeckhaute, "Performance of emerging multi-carrier waveforms for 5G asynchronous communications," EURASIP J. Wirel. Commun. Netw., vol. 2017, no. 1, pp. 1-15, 2017.

[10] G. Fettweis, M. Krondorf, and S. Bittner, "GFDM - Generalized frequency division multiplexing," IEEE Veh. Technol. Conf., pp. 1-4, 2009.

[11] L. Chrislin and D. R. Le, "Decoding Schemes for FBMC with Single-Delay STTC," EURASIP Journal on Advances in Signal Processing, vol. 2010, 2010.

[12] A. Viholainen, T. Thalainen, T. H. Stitz, M. Renfors, and M. Bellanger, "Prototype filter design for filter bank based multicarrier transmission,” Eur. Signal Process. Conf., no. Eusipco, pp. 1359-1363, 2009.

[13] R. N. M. Rupp, "On Pilot-Symbol Aided Channel Estimation in Fbmc-Oqam Ronald Nissel Markus Rupp Technische Universit " at Wien , Institute of Telecommunications Gusshausstraße 25 , 1040 Vienna , Austria," Icassp 2016, pp. 3681-3685, 2016.

[14] M. Mukherjee, L. Shu, V. Kumar, P. Kumar, and R. Matam, "Reduced out-of-band radiation-based filter optimization for UFMC systems in 5G," in IWCMC 2015 - 11th International Wireless Communications and Mobile Computing Conference, 2015, pp. 1150-1155.

[15] M. El Hefnawy and H. Taoka, "Overview of Faster-Than-Nyquist for Future Mobile Communication Systems," in IEEE 77th Vehicular Technology Conference (VTC Spring), 2013, 2013.

[16] J. E. Mazo, "Faster-Than-Nyquist Signaling,” Bell Syst. Tech. J., vol. 54, no. 2, pp. 1451-1462, 1975.

[17] J. B. Anderson, "A Review of Faster than Nyquist Signaling with an Extension to Four-Level Modulation," in 2016 9th International Symposium on Turbo Codes \& Iterative Information Processing, 2016, pp. 6-10.

[18] M. Hamamura and S. Tachikawa, "Bandwidth efficiency improvement for multi-carrier systems," in 15th IEEE International Symposium on Personal, Indoor and Mobile Radio Communications, PIMRC 2004., 2004, vol. 1, pp. 48-52.

[19] S. I. A. Ahmed, "Spectrally Efficient FDM Communication Signals and Transceivers : Design , Mathematical Modeling and System Optimization by Statement of Originality," 2011.

[20] M. R. D. Rodrigues and I. Darwazeh, "A spectrally efficient frequency division based communications system," Proc. Int. Symp. Broadband Commun., no. September, 2006.

[21] S. Isam and I. Darwazeh, "Simple DSP-IDFT Techniques for Generating Spectrally Efficient FDM Signals," in 7th International Symposium on Communication Systems Networks and Digital Signal Processing (CSNDSP), 2010, 
2010, pp. 20-24.

[22] J. Wang, X. Yang, X. H. Zhang, and D. Li, "The prefix design and performance analysis of DFT-based overlapped frequency division multiplexing (OvFDM-DFT) system," IWSDA 07 - 3rd Int. Work. Signal Des. Its Appl. Commun., no. Ic, pp. 361-364, 2007.

[23] I. Kanaras, A. Chorti, M. R. D. Rodrigues, and I. Darwazeh, "A combined MMSE-ML detection for a spectrally efficient non orthogonal FDM signal," 5th Int. Conf. Broadband Commun. Networks, Syst. BROADNETS 2008, vol. 1, no. 3, pp. 421-425, 2008.

[24] S. Hong and J. Seo, "Cyclic prefix / suffix-assisted Frequency-Domain Equalization for Faster-than-Nyquist Signaling Block Transmission," in 2016 IEEE 27th Annual IEEE International Symposium on Personal, Indoor and Mobile Radio Communications - (PIMRC): Fundamentals and PHY, 2016.

[25] N. Ahmad, S. K. Syed-Yusof, N. Fisal, K. Anwar, and T. Matsumoto, "Soft-feedback MMSE equalization for nonorthogonal frequency division multiplexing (n-OFDM) signal detection," in 2012 16th International ITG Workshop on Smart Antennas, WSA 2012, 2012, pp. 248-255.

[26] E. Bedeer, M. H. Ahmed, and H. Yanikomeroglu, "Low-Complexity Detection of High-Order QAM Faster-ThanNyquist Signaling," IEEE Access, vol. 5, pp. 14579-14588, 2017.

[27] I. M. B. Mokhtar, N. Ahmad, H. M. Kaidi, M. A. M. Izhar, and M. N. Mohamed, "PAPR reduction techniques in generalized inverse discrete fourier transform non-orthogonal frequency division multiplexing system," Indones. J. Electr. Eng. Comput. Sci., vol. 10, no. 3, pp. 1045-1052, 2018.

[28] J. Hagenauer, "The exit chart - Introduction to extrinsic information transfer in iterative processing," in European Signal Processing Conference, 2015, vol. 06-10-Sept, pp. 1541-1548.

\section{BIOGRAPHIES OF AUTHORS}
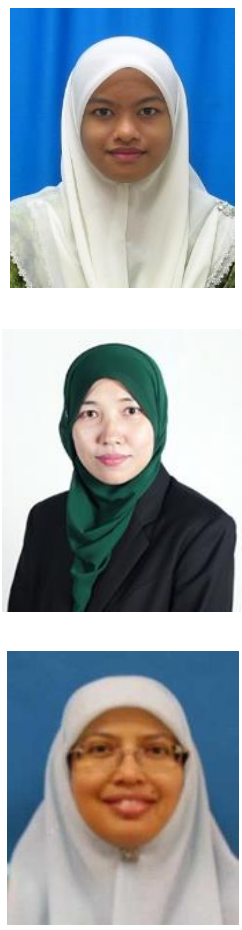

Siti Rosmaniza Ab Rashid is a Ph.D. Student in the School of Electrical Engineering at Universiti Teknologi Malaysia. She can be reached at sitirosmaniza@utem.edu.my.

Dr. Norulhusna Ahmad is a Senior Lecturer at Razak Faculty of Technology and Informatics, Universiti Teknologi Malaysia Kuala Lumpur. Her expertise is in the area of digital signal processing and wireless communication. Her research interests are on future communication such as $5 \mathrm{G}$ and cognitive radio focusing on error-correcting codes, turbo equalization, OFDM, resource allocation, network coding, and cooperative communication. She can be reached at norulhusna.kl@utm.my.

Dr. Sharifah Kamilah is an Associate Professor in the School of Electrical Engineering at Universiti Teknologi Malaysia. Her expertise is in the area of digital wireless communication. Her research interests are on the OFDM-based system, software defined radio, cognitive radio system, software-defined network, network coding, cooperative communication, and quality management system. She can be reached at kamilah@utm.my. 